\title{
Mitigating optical losses in crystalline silicon thin-film solar cells on glass
}

\author{
David Eisenhauer, Bernd Rech, and Christiane Becker \\ Helmholtz-Zentrum Berlin für Materialien und Energie GmbH, 12489 Berlin, Germany
}

\begin{abstract}
Liquid phase crystallized silicon thin-film solar cells on glass provide efficiencies up to $14.2 \%$. While open-circuit voltage and fill factor are already comparable to wafer-based devices, short-circuit current density is reduced due to incomplete light absorption. This paper analyzes the losses of current device designs in experiment and one-dimensional simulations, revealing the low absorber thickness of $15-20 \mu \mathrm{m}$ as well as the planar glass-silicon interface as the main cause of non-absorption. Interface textures, in particular a sinusoidal texture and a smooth anti-reflective three-dimensional (SMART) texture, are discussed concerning their potential to mitigate these losses, allowing to reduce losses at the glass-silicon interface by at least $40 \%$ relative. Taking the electronic interface quality into account, the SMART texture is identified as the most promising texture for light management in liquid phase crystallized silicon thin-film solar cells on glass.
\end{abstract}

Index Terms - silicon, thin-film solar cells, light management, absorption enhancement.

\section{INTRODUCTION}

Liquid phase crystallization (LPC) of $15-20 \mu \mathrm{m}$ thick silicon layers is a promising technology for thin-film solar cells with both, high efficiencies and low costs. Recently, a power conversion efficiency of $14.2 \%$ could be demonstrated [1], whereby the open-circuit voltage of $654 \mathrm{mV}$ and fill factor of $75 \%$ are already close to values achieved of record devices for wafer-based high-performance multi-crystalline silicon solar cells [2]. The lower efficiency mainly results from a reduced short-circuit current density caused by optical losses. These losses arise from the more than tenfold lower absorber thickness of the LPC silicon solar cell and high reflectance at planar interfaces. Reducing the losses requires improved light management in order to increase the short-circuit current density in LPC silicon thin-film solar cells on glass and in order to draw closer to efficiencies of wafer-based approaches. One method that has proven to yield an increased light incoupling into the absorber is the introduction of textures at the glass-silicon interface, both using random [3]-[5] and periodic [6]-[8] structures. However, a trade-off between improved optical properties from pronounced texturing and deteriorated electronic material quality due to texture-induced interface defects was identified [6], posing further requirements for suitable light management schemes. Texturing methods that allowed both, improving light in-coupling while preserving an electronic material quality comparable to planar LPC devices include a sinusoidal texture [7] and a smooth anti-reflective three-dimensional (SMART) texture [8], as demonstrated by maximum open-circuit voltages of $618 \mathrm{mV}$ and $649 \mathrm{mV}$, respectively.

In this contribution, we discuss the optical losses of the stateof-the-art device design of liquid phase crystallized silicon thin-film solar cells on glass with a planar glass-silicon interface in experiment and one-dimensional simulations. Based on these results, the potential gains from glass-silicon texturing are presented considering both optical properties as well as the influence of interface texturing on interface and bulk material properties. From these, an optically and electronically optimized light management scheme is derived and a horizon for the power conversion efficiency of textured LPC silicon thin-film solar cells on glass is given.

\section{EXPERIMENTAL AND NUMERICAL METHODS}

\section{A. Sample Preparation}

Liquid phase crystallized silicon thin-film solar cells on glass substrates were produced with a planar interlayer stack consisting of a $250 \mathrm{~nm}$ thick silicon oxide $\left(\mathrm{SiO}_{\mathrm{x}}\right) / 60 \mathrm{~nm}$ silicon nitride $\left(\mathrm{SiN}_{\mathrm{x}}\right) / 10 \mathrm{~nm}$ silicon oxy-nitride $(\mathrm{Si}(\mathrm{ON}))$ serving as diffusion barrier, anti-reflective coating, and diffusion and wetting layer, respectively, between glass substrate and silicon absorber [1], [9]. Additionally to this planar reference interlayer stack, two interlayer textures were investigated in this study, namely a sinusoidal texture [7] and a smooth antireflective three-dimensional (SMART) texture [8]. The hexagonal sinusoidal texture exhibits a period of $750 \mathrm{~nm}$ and a height-to-period ratio of 0.5 and is produced by combining nano-imprint lithography into a commercially available organic resist with reactive ion etching to replicate the texture in the $\mathrm{SiO}_{x}$ diffusion barrier [10]. $\mathrm{SiN}_{\mathrm{x}} / \mathrm{Si}(\mathrm{ON})$ interlayers were subsequently deposited on the sinusoidal texture. For the

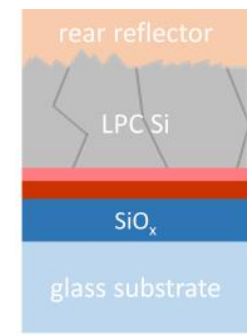

Reference
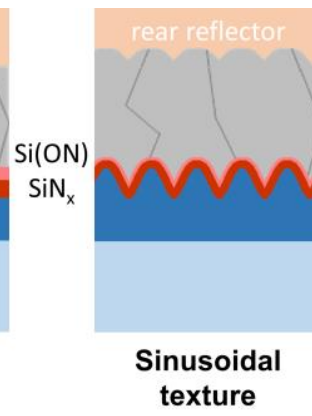

$\mathrm{SiO}_{\mathrm{x}}$ $\mathrm{TiO}_{\mathrm{x}}$

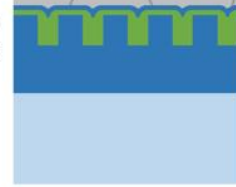

SMART texture
Fig. 1. Schematic sample stacks of the samples investigated (not to scale). 
SMART texture, a hexagonal nano-pillar array with a period of $750 \mathrm{~nm}$ and height of $\sim 60 \mathrm{~nm}$ replicated in hightemperature stable sol-gel and subsequently smoothed by spincoating of a titanium oxide $\left(\mathrm{TiO}_{\mathrm{x}}\right)$ precursor solution. Thermal annealing for $30 \mathrm{~min}$ at $800^{\circ} \mathrm{C}$ results in a compact crystalline $\mathrm{TiO}_{\mathrm{x}}$ layer, substituting the silicon nitride anti-reflective coating. The spin-coated $\mathrm{TiO}_{\mathrm{x}}$ preferably fills the voids between the nano-pillars, leading to an "optically rough" texture with a morphologically smooth surface. A $10 \mathrm{~nm}$ thick $\mathrm{SiO}_{\mathrm{x}}$ layer is employed as wetting and diffusion barrier. All samples were coated with $15 \mu \mathrm{m}$ thick silicon absorbers by electron-beam evaporation, followed by a $100 \mathrm{~nm}$ thick $\mathrm{SiO}_{\mathrm{x}}$ capping layer.

Liquid phase crystallization is performed using a lineshaped laser with a width of $5 \mathrm{~cm}$ and a wavelength of $808 \mathrm{~nm}$. Rapid thermal annealing for $1 \mathrm{~min}$ at $950{ }^{\circ} \mathrm{C}$ after $\mathrm{LPC}$ reduces thermal stress in the glass substrate. The capping layer is removed by wet-chemical etching in a buffered oxide etch solution for $9 \mathrm{~min}$. The top $\sim 300 \mathrm{~nm}$ of the LPC silicon layer is etched away in a silicon etching solution consisting of a hydrous solution of hydrofluoric acid, nitric acid and phosphorous acid for $1 \mathrm{~min}$. While the sinusoidal texture exhibits a double-sided texturing due to the usage of a capping layer [11], a random pyramid back-side texture is introduced for the reference and SMART texture samples by wetchemical etching in an IPA-free solution containing potassium hydroxide (Alkatex free provided by GP Solar) for $3 \mathrm{~min}$ at $80^{\circ} \mathrm{C}$. For the SMART texture, an additional sample with $\mathrm{Si}(\mathrm{ON})$ passivation layer is produced by solid phase crystallization for $20 \mathrm{~h}$ at $600{ }^{\circ} \mathrm{C}$, as so far no successful liquid phase crystallization was feasible using this interlayer combination. Solar cells were produced using the lithographyfree contacting scheme introduced by Haschke et al. [12], therein denoted as test cells. All samples were equipped with a white-paint rear reflector. Figure 1 schematically illustrates the samples investigated. Absorptance measurements were performed using a Perkin Elmer Lambda 1050 photospectrometer holding the samples in the center of an integrating sphere with a diameter of $15 \mathrm{~cm}$. External quantum efficiency is measured using a home-made set-up with a spot size of $3 \times 2 \mathrm{~mm}^{2}$. Atomic force microscope images used as input for simulations were obtained using a Park Systems XE70.

\section{B. Simulations}

One-dimensional simulations were carried out using the optical simulation software "GenPro4" developed at Delft University of Technology [13]. The software code numerically calculates the absorptance in a layer stack based on the transfer matrix method. Textured interfaces can be treated despite the one-dimensional simulation approach by the implementation of a ray tracing and scalar scattering model [14]. The ray tracing model is employed for the random pyramid texture, for which surface topography data determined by atomic force microscope measurements were used as input. Refractive indices of the layers were taken from literature in case of crystalline silicon [15] or measured by spectroscopic ellipsometry.

\section{RESULTS}

\section{A. Loss Analysis of Planar LPC Silicon Absorbers}

In order to assess the potential of textures at the glasssilicon interface to reduce optical losses in LPC silicon solar cells on glass, losses of the planar state-of-the-art device design were identified as a first step. Figure 2 depicts the measured absorptance in a $15 \mu \mathrm{m}$ thick LPC silicon absorber (black curve) as well as the calculated absorptance (grey area) from one-dimensional simulations. The curves match well, with only minor deviations for wavelengths $>400 \mathrm{~nm}$, which are attributed to inaccuracies in modelling and, supposedly, defect absorption in the long wavelength range of the real structure. In the short wavelength range up to $400 \mathrm{~nm}$, absorptance of the measured sample rises due to parasitic absorption in the glass substrate, which had not been considered in the simulations.

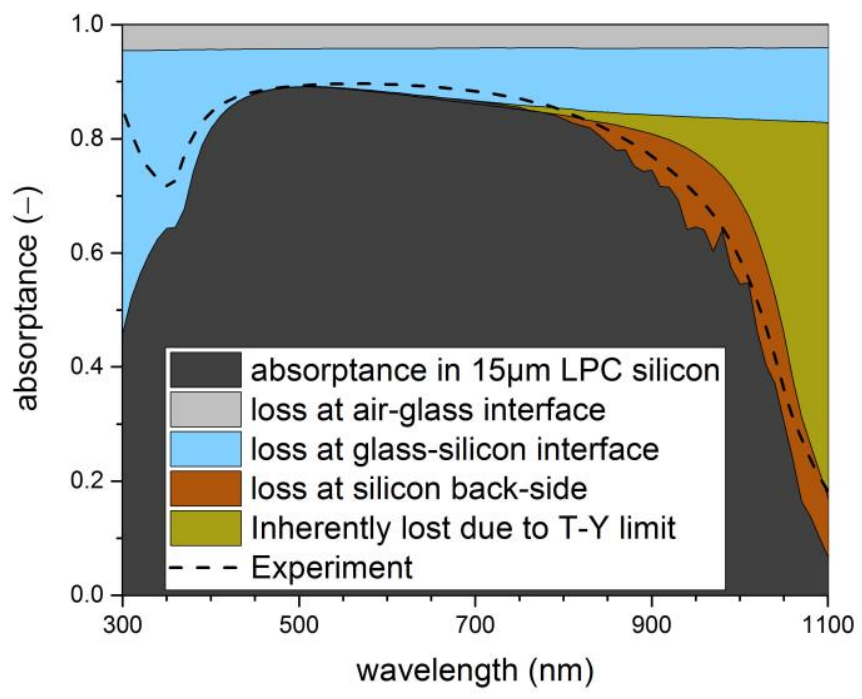

Fig. 2. Absorptance in $15 \mu \mathrm{m}$ thick LPC silicon from measurement (black dashed line) and one-dimensional simulations (grey area). Losses stemming from the air-glass interface (light grey), glasssilicon interface (blue) and silicon back-side (brown) are displayed. Losses related to the Tiedje-Yablonovitch (T-Y) limit for a $15 \mu \mathrm{m}$ thick silicon layer considering reflectance at the air-glass and glasssilicon interface are included for reference (dark yellow area).

One-dimensional simulations allow to easily adapt the structure under consideration. In particular, specific interfaces can be "switched off", e.g. neglecting the rear-side of the silicon absorber by assuming an infinitely thick absorber. In doing so, losses can be attributed to a specific interface, as illustrated by differently colored areas in Fig. 2 . The air-glass interface (light grey area) causes a loss of around $4 \%$ of the 
incoming light independent of wavelength. This amounts to an equivalent maximum achievable short-circuit current density loss of $1.8 \mathrm{~mA} \mathrm{~cm}^{-2}$. The glass-silicon interface (blue area) has a minimum reflectance of around $6 \%$ at a wavelength of $500 \mathrm{~nm}$. For longer wavelength, reflectance slowly increases up to $15 \%$ for $1100 \mathrm{~nm}$. In the short wavelength range, a steep increase to around $50 \%$ is observed. In total, an equivalent of $4.5 \mathrm{~mA} \mathrm{~cm}^{-2}$ is reflected at this interface. The rear interface only plays a role for wavelengths $>700 \mathrm{~nm}$, for which the penetration depth of the incoming light becomes larger than the absorber thickness. Due to the relatively low absorption coefficient of silicon in the near infrared, a large proportion of the incident light is lost due to non-absorption after passing through the absorber. As a benchmark for light trapping, the Tiedje-Yablonovitch limit [16] for a $15 \mu \mathrm{m}$ thick silicon layer considering reflectance at the air-glass and glass-silicon interface (dark yellow area) is included in Fig. 2. In this limit, non-absorption steeply rises for wavelengths $>900 \mathrm{~nm}$ and an equivalent of $3.4 \mathrm{~mA} \mathrm{~cm}^{-2}$ is lost. Hence, optical losses arising from the rear-side of the silicon layer amount to $0.9 \mathrm{~mA} \mathrm{~cm}^{-2}$ (brown area) if the limitations imposed by the TiedjeYablonovitch limit are considered.

Overall, the current device design of LPC silicon thin-film solar cells on glass leads to losses of the maximum achievable short-circuit current density of $7.1 \mathrm{~mA} \mathrm{~cm}^{-2}$ compared to the Tiedje-Yablonovitch limit for $15 \mu \mathrm{m}$ silicon. Of these, $4.5 \mathrm{~mA} \mathrm{~cm}^{-2}$ can be attributed to the planar glass-silicon interface, proving the necessity of interface textures at this interface.

\section{B. Loss Analysis of Textured LPC Silicon Absorbers}

Both, the sinusoidal texture [7], [10] and SMART texture, [8] have demonstrated improved light in-coupling into the silicon absorber. Figure 3 exhibits the absorptance of $15 \mu \mathrm{m}$ thick LPC silicon absorbers with a planar glass-silicon interface (black), a sinusoidal texture (red) and a SMART texture (green). For the SMART texture, two passivation layers were employed, namely silicon oxide (solid curve) and silicon oxy-nitride (dashed). The analysis of the sample with $\mathrm{Si}(\mathrm{ON})$ passivation is restricted to the wavelength range $300 \mathrm{~nm}-600 \mathrm{~nm}$ to exclude effects from the flat back-side of the SPC silicon sample. Reflectance at the air-glass interface (grey area) and the Tiedje-Yablonovitch limit considering reflectance at the air-glass interface (T-Y limit*(1-R)air-glass, dotted) are added for reference to constitute a benchmark for reflectance at the glass-silicon interface.

Both textures efficiently improve anti-reflective properties of LPC silicon absorbers on glass. Comparing the passivation layers for the SMART texture reveal the influence of the $10 \mathrm{~nm}$ thin top layer on optical properties. The silicon oxide layer causes an increased reflectance in the short-wavelength range due to its lower refractive index. The SMART texture has a minimum reflectance of $5 \%$ at $650 \mathrm{~nm}$, of which $4 \%$ are reflected at the air-glass interface. For the reference sample, a

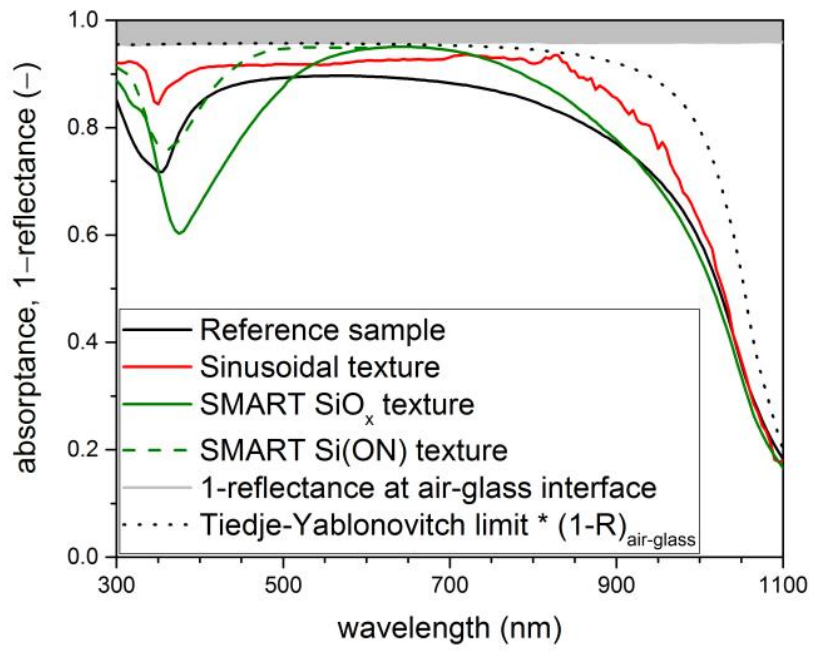

Fig. 3. Absorptance in $15 \mu \mathrm{m}$ thick LPC silicon absorbers with a planar glass-silicon interface (black), exhibiting a sinusoidal texture (red), and a SMART texture (green). For the SMART texture, both a silicon oxide $\left(\mathrm{SiO}_{\mathrm{x}}\right.$, solid curve) and silicon oxy-nitride $(\mathrm{Si}(\mathrm{ON})$, dashed) passivation layer is depicted. Reflectance at the air-glass interface represented as 1-R (grey area) and Tiedje-Yablonovitch limit after glass reflectance (dotted) is added for reference.

minimum reflectance of $10 \%$ is found. While the sinusoidal texture exhibits a maximum of $7 \%$ reflectance, it provides broader anti-reflective properties compared to the SMART texture, minimizing reflectance in the wavelengths range $300 \mathrm{~nm}-400 \mathrm{~nm}$ and $750 \mathrm{~nm}-1000 \mathrm{~nm}$. Total losses compared to the solar irradiance of the textured samples amount to $7.2 \mathrm{~mA} \mathrm{~cm}^{-2}$ and $8.9 \mathrm{~mA} \mathrm{~cm}^{-2}$ for the LPC silicon absorber with a sinusoidal texture and SMART texture with $\mathrm{SiO}_{\mathrm{x}}$ passivation layer, respectively.

Replacing the $\mathrm{SiO}_{x}$ passivation layer with $\mathrm{Si}(\mathrm{ON})$ in an $\mathrm{LPC}$ absorber is expected to reduce losses in the SMART texture devices by an additional $1.0 \mathrm{~mA} \mathrm{~cm}^{-2}$ due to lower reflectance in the short wavelength range. Thus, total losses of $7.9 \mathrm{~mA} \mathrm{~cm}^{-}$ 2 may be reached for SMART textured LPC devices with $\mathrm{Si}(\mathrm{ON})$ passivation layer. Of these losses, $1.8 \mathrm{~mA} \mathrm{~cm}^{-2}$ are reflected at the air-glass interface and $3.4 \mathrm{~mA} \mathrm{~cm}^{-2}$ are inherently lost, as calculated from the Tiedje-Yablonovitch limit. Hence, the textured glass-silicon interface cause losses of $2.0 \mathrm{~mA} \mathrm{~cm}^{-2}$ and $2.7 \mathrm{~mA} \mathrm{~cm}^{-2}$, a $56 \%$ and $40 \%$ relative gain compared to the state-of-the-art device design for the sinusoidal and SMART texture with $\mathrm{Si}(\mathrm{ON})$ passivation layer.

\section{External Quantum Efficiency}

Improved light management arising from interface texturing will only lead to an efficiency enhancement if the texture does not affect interface or bulk material quality of the solar cell.

Hence, measurements of the external quantum efficiency (EQE) were performed to further analyze the potential of textures at the glass-silicon interface of LPC silicon thin-film solar cells on glass. Internal quantum efficiency (IQE) was 
calculated from absorptance and external quantum efficiency measurements. Figure 4 displays the EQE (solid) and IQE (dashed) of LPC silicon solar cells with a planar glass-silicon interface (black), a sinusoidal texture (red) and a SMART texture with $\mathrm{SiO}_{x}$ passivation layer (green). The reference sample exhibits an EQE of approximately $80 \%$ in the wavelength range from $400 \mathrm{~nm}$ to $800 \mathrm{~nm}$, corresponding to an IQE of $90 \%$. For longer wavelengths, EQE drops in line with absorptance.

For the solar cell with a SMART texture, IQE is slightly lower compared to the solar cell with planar glass-silicon interface.

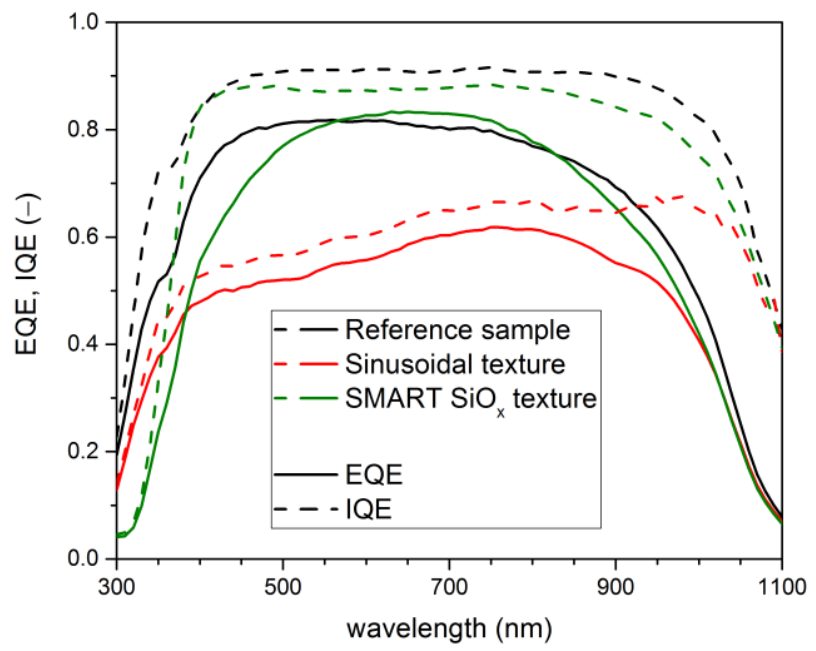

Fig. 4. External (solid) and internal (dashed) quantum efficiency (solid) of LPC silicon solar cells with a planar glasssilicon interface (black), exhibiting a sinusoidal texture (red), and a SMART texture with $\mathrm{SiO}_{x}$ passivation layer (green).

This may be explained by a lower passivation quality provided by the interlayer stack, in particular the silicon oxide passivation layer compared to its silicon oxy-nitride counterpart. Nonetheless, an IQE exceeding $80 \%$ is achieved for the solar cell produced on a SMART textured substrate. EQE of the sample with a sinusoidal texture with height-toperiod ratio of 0.5 , on the contrary, reveals an inferior EQE and IQE limited to values below $70 \%$. This is attributed to interface defects induced by the texture occurring despite the optimized interlayer stack that demonstrated surface recombination velocities as low as $200 \mathrm{~cm} \mathrm{~s}^{-1}$ for planar devices [1].

\section{DISCUSSION AND CONCLUSION}

Liquid phase crystallized silicon thin-film absorbers were analyzed in regards to their optical properties. Onedimensional simulations allow to differentiate losses stemming from different interfaces in the device. The glass-silicon interface could thereby be identified as the most important one, with a loss of $4.5 \mathrm{~mA} \mathrm{~cm}{ }^{-2}$ out of $7.1 \mathrm{~mA} \mathrm{~cm}^{-2}$ compared to the Tiedje-Yablonovitch limit for a $15 \mu \mathrm{m}$ thick silicon layer. Losses caused by the air-glass interface and silicon back-side amount to $1.8 \mathrm{~mA} \mathrm{~cm}^{-2}$ and $0.8 \mathrm{~mA} \mathrm{~cm}^{-2}$, respectively.

Sinusoidal textures and SMART textures were implemented at the glass-silicon interface to mitigate these losses. For the SMART texture, two passivation layers were investigated. Employing these measures, the losses at this interface were reduced from $4.5 \mathrm{~mA} \mathrm{~cm}^{-2}$ for a planar interlayer stack to $2.0 \mathrm{~mA} \mathrm{~cm}{ }^{-2}$ for a sinusoidal texture with height-to-period ratio of 0.5 , and $3.7 \mathrm{~mA} \mathrm{~cm}^{-2}$ and $2.7 \mathrm{~mA} \mathrm{~cm}^{-2}$ for a SMART texture with silicon oxide and with silicon oxy-nitride passivation layer, respectively.

Internal quantum efficiencies well above $80 \%$ were measured on solar cells with a planar interlayer stack and SMART texture with silicon oxide passivation layer. For the sinusoidal nano-texture, a detrimental effect on interface and bulk material quality lead to a reduction of IQE to below $70 \%$. Consequently, the most promising light management scheme combining both, improved optical properties and interface and bulk properties equivalent to devices with planar glass-silicon interface, comprises a SMART texture at the glass-silicon interface and a random pyramid texture at the rear side of the absorber produced by wet-chemical etching in $\mathrm{KOH}$. To fully exploit the optical potential of the SMART texture, it requires to be combined with a silicon oxy-nitride passivation layer. In addition, anti-reflective measures at the air-glass interface, e.g. a moth-eye texture, may contribute to reducing optical losses by reducing reflectance from $4 \%$ to less than $1 \%$. In terms of short-circuit current density, losses at this interface may thereby be lowered to $0.4 \mathrm{~mA} \mathrm{~cm}^{-2}$.

Combining all these measures, maximum achievable shortcircuit current density may be enhanced from $33.4 \mathrm{~mA} \mathrm{~cm}^{-2}$ to $36.8 \mathrm{~mA} \mathrm{~cm}^{-2}$. Assuming an IQE of 0.9 , a short-circuit current density of $33.4 \mathrm{~mA} \mathrm{~cm}^{-2}$ is feasible. If this may be combined with an open-circuit voltage and fill factor already reached on individual solar cells to $670 \mathrm{mV}$ and $79 \%$, respectively, a power conversion efficiency of $18 \%$ is feasible for LPC silicon thin-film solar cells on glass.

\section{ACKNOWLEDGEMENT}

The German ministry of education and research (BMBF) is acknowledged for funding the research activities of the Young Investigator Group 'Nano-SIPPE' at HZB in the program NanoMatFutur (no. 03X5520). The authors thank Dr. Klaus Jäger and Dr. Daniel Amkreutz for fruitful discussions. GP Solar is acknowledged for providing the Alkatex IPA-free texturing agent.

\section{REFERENCES}

[1] C. Thi Trinh et al., "Potential of interdigitated back-contact silicon heterojunction solar cells for liquid phase crystallized silicon on glass with efficiency above 14\%," Sol. Energy Mater. Sol. Cells, vol. 174, no. June 2017, pp. 187-195, 2018. 
[2] J. Benick et al., "High-Efficiency n-Type HP mc Silicon Solar Cells," IEEE J. Photovoltaics, vol. 7, no. 5, pp. 11711175, 2017.

[3] M. Z. Pakhuruddin, J. Dore, J. Huang, and S. Varlamov, "Effects of front and rear texturing on absorption enhancement in laser-crystallized silicon thin-films on glass,” Jpn. J. Appl. Phys., vol. 54, p. 08KB04, 2015.

[4] M. Z. Pakhuruddin, J. Huang, J. Dore, and S. Varlamov, "Enhanced light-trapping in laser-crystallised silicon thinfilm solar cells on glass by optimised back surface reflectors," Sol. Energy, vol. 150, pp. 477-484, 2017.

[5] G. Köppel et al., "Periodic and Random Substrate Textures for Liquid-Phase Crystallized Silicon Thin-Film Solar Cells," IEEE J. Photovoltaics, vol. 7, no. 1, pp. 85-90, 2017.

[6] V. Preidel, D. Amkreutz, J. Haschke, M. Wollgarten, B. Rech, and C. Becker, "Balance of optical, structural, and electrical properties of textured liquid phase crystallized Si solar cells," J. Appl. Phys., vol. 117, p. 225306, 2015.

[7] G. Köppel, B. Rech, and C. Becker, "Sinusoidal nanotextures for light management in silicon thin-film solar cells," Nanoscale, vol. 8, no. 16, pp. 8722-8728, 2016.

[8] D. Eisenhauer et al., "Smooth anti-reflective threedimensional textures for liquid phase crystallized silicon thin-film solar cells on glass," Sci. Rep., vol. 7, p. 2658, 2017.

[9] N. Preissler et al., "Impact of Dielectric Layers on LiquidPhase Crystallized Silicon Solar Cells," IEEE J. Photovoltaics, vol. 8, no. 1, pp. 30-37, 2018.
[10] D. Eisenhauer, G. Köppel, B. Rech, and C. Becker, "Improved light management in crystalline silicon thin-film solar cells by advanced nano-texture fabrication," in Light, Energy and the Environment, 2017, p. PW2A.4.

[11] C. Becker, V. Preidel, D. Amkreutz, J. Haschke, and B. Rech, "Double-side textured liquid phase crystallized silicon thin-film solar cells on imprinted glass," Sol. Energy Mater. Sol. Cells, vol. 135, pp. 2-7, 2015.

[12] J. Haschke, D. Amkreutz, L. Korte, F. Ruske, and B. Rech, "Towards wafer quality crystalline silicon thin-film solar cells on glass," Sol. Energy Mater. Sol. Cells, vol. 128, pp. 190-197, 2014.

[13] R. Santbergen, A. H. M. Smets, and M. Zeman, "Optical model for multilayer structures with coherent, partly coherent and incoherent layers.," Opt. Express, vol. 21, pp. A262-A267, 2013.

[14] K. Jäger, M. Fischer, R. A. C. M. M. Van Swaaij, and M. Zeman, "A scattering model for nano-textured interfaces and its application in opto-electrical simulations of thin-film silicon solar cells," J. Appl. Phys., vol. 111, no. 8, 2012.

[15] M. A. Green, "Self-consistent optical parameters of intrinsic silicon at $300 \mathrm{~K}$ including temperature coefficients," Sol. Energy Mater. Sol. Cells, vol. 92, no. 11, pp. 1305-1310, Nov. 2008.

[16] T. Tiedje, E. Yablonovitch, G. D. Cody, and B. G. Brooks, "Limiting efficiency of silicon solar cells," IEEE Trans. Electron Devices, vol. 31, no. 5, pp. 711-716, 1984. 